\title{
Entanglement Swapping with Semiconductor-Generated Photons Violates Bell's Inequality
}

\author{
Michael Zopf $\odot,{ }^{1}$ Robert Keil, ${ }^{1}$ Yan Chen, ${ }^{1}$ Jingzhong Yang, ${ }^{1,2}$ Disheng Chen, ${ }^{1}$ \\ Fei Ding, ${ }^{1,2, *}$ and Oliver G. Schmidt ${ }^{1,3, *}$ \\ ${ }^{1}$ Institute for Integrative Nanosciences, Leibniz, IFW Dresden, Helmholtzstraße 20, 01069 Dresden, Germany \\ ${ }^{2}$ Institut für Festkörperphysik, Leibniz Universität Hannover, Appelstraße 2, 30167 Hannover, Germany \\ ${ }^{3}$ Material Systems for Nanoelectronics, Technische Universität Chemnitz, 09107 Chemnitz, Germany
}

(Received 4 March 2019; published 14 October 2019)

\begin{abstract}
Transferring entangled states between photon pairs is essential in quantum communication. Semiconductor quantum dots are the leading candidate for generating polarization-entangled photons deterministically. Here we show for the first time swapping of entangled states between two pairs of photons emitted by a single dot. A joint Bell measurement heralds the successful generation of the Bell state $\Psi^{+}$, yielding a fidelity of $0.81 \pm 0.04$ and violating the CHSH and Bell inequalities. Our photon source matches atomic quantum memory frequencies, facilitating implementation of hybrid quantum repeaters.
\end{abstract}

DOI: 10.1103/PhysRevLett.123.160502

Semiconductor light sources have revolutionized science and technology since laser diodes [1,2] and vertical-cavity surface-emitting lasers (VCSELs) [3,4] arrived in the 1960s. Quantum mechanics lies at the roots for these devices, yet quantum states of light have only been studied extensively in recent decades in their own right—sparking the "second quantum revolution." Semiconductor sources can now emit single photons $[5,6]$ and entangled photons [7] on demand, more reliably and intensely than nonlinear crystals. They hold great potential for a range of applications in quantum communication [8], quantum metrology [9], and quantum computation [10].

The next step towards building quantum networks is to transfer entangled states between distinct pairs of photons [11-13]. This entails substituting the pairwise entanglement in two-photon states with entanglement between photons from different pairs $[14,15]$. The first experiment to do this, two decades ago [16], used a technique based on spontaneous parametric downconversion in a nonlinear optical crystal [17-19]. Though such sources are widely used, for example, to entangle multiple photons [20], their brightness and therefore scalability are fundamentally limited owing to Poissonian emission statistics [21].

Semiconductor quantum dots (QDs), by contrast, are able to generate entangled photon pairs deterministically one by one [22]. However, until recently, QDs were too faint and of poor degree of entanglement and indistinguishability to use for advanced quantum applications. Improvements of the past three years have overcome these limitations. Highly coherent [23] and strongly entangled photons $[24,25]$ can now be generated with high brightness [26] and reproducibility [24] from QDs.

Here we demonstrate, for the first time, entanglement swapping between polarization-entangled photons emitted by a semiconductor QD. The Bell state $\Psi^{+}$is generated with high fidelity and strong nonlocal characteristics, proven by violating the $\mathrm{CHSH}$ and Bell inequalities $[27,28]$. Our semiconductor sources are emitting at atomic quantum memory frequencies. This promotes their use in devices such as quantum repeaters (the quantum equivalent of a classical amplifier) [29], which are essential for longdistance quantum communication.

The experimental concept is sketched in Fig. 1. Two pairs of polarization-entangled photons are consecutively emitted (emissions 1 and 2) by a single semiconductor QD. The polarization of one photon from each pair is measured by separate detectors, labeled Alice and Bob. Then, a joint Bell state measurement (BSM) is made on the remaining two photons; this swaps the entanglement of the original pairs to the photons that Alice and Bob receive. The bright

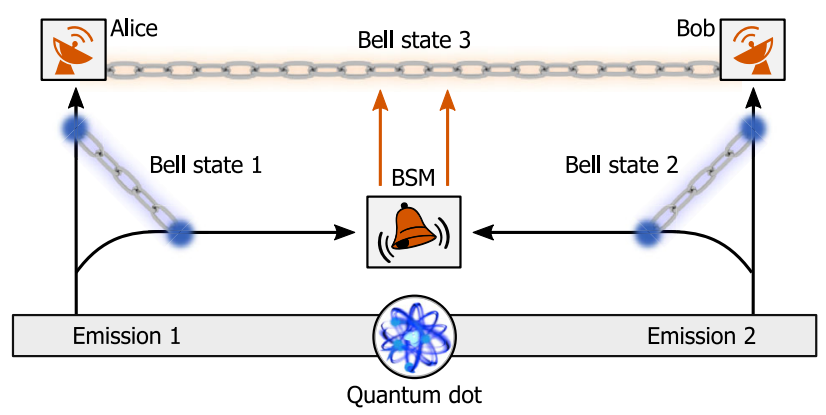

FIG. 1. Principle of entanglement swapping with polarizationentangled photons emitted by a single quantum dot. Two entangled photon pairs are generated (emissions 1 and 2). One photon from each pair is directed to a BSM. Upon success, the BSM establishes entanglement of the remaining photons sent to Alice and Bob. 

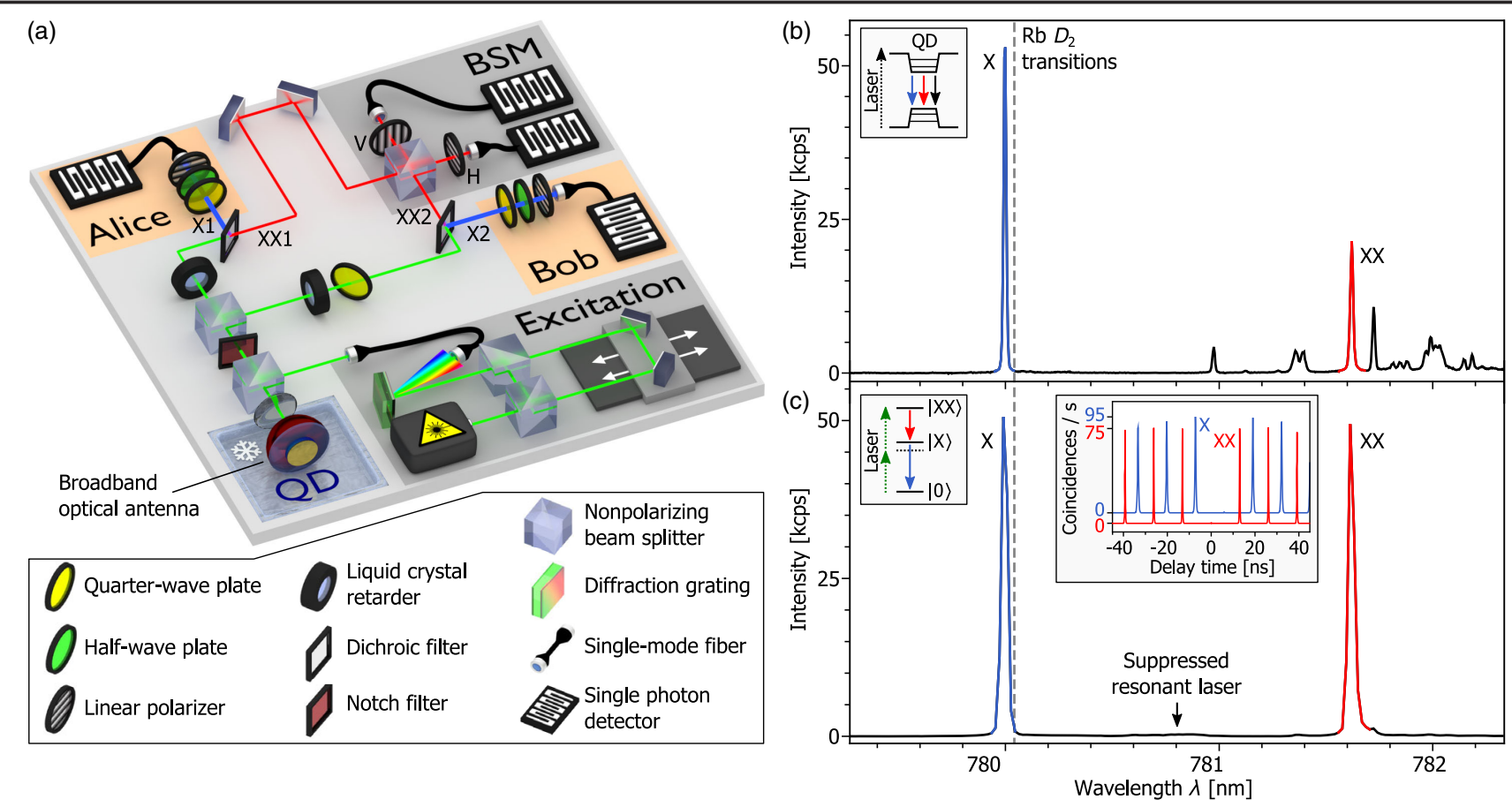

FIG. 2. Experimental setup and quantum dot emission spectra. (a) Entanglement swapping setup. Two consecutive pairs of polarization-entangled photons $\mathrm{X} i-\mathrm{XX} i$ (emission $i=1,2$ ) are generated by a QD. Two photons XX1 and XX2 from each emission are directed to a Bell state measurement. Coincidence detection heralds the polarization entanglement of the remaining photons X1 and X2. The latter are guided to two polarization analyzers, Alice and Bob. Liquid crystal retarders and a quarter-wave plate are used to compensate for birefringence in the setup. (b) QD photoluminescence spectrum under above-band-gap excitation highlighting the prominent $\mathrm{X}$ and $\mathrm{XX}$ emissions. (c) Emission spectrum under pulsed resonant two-photon excitation of the biexciton state. Decay via the intermediate exciton states results in the emission of polarization-entangled photon pairs XX-X. The inset shows the intensity autocorrelations, indicating a high single-photon purity of $g^{(2)}(0) \leq 0.005$.

sources of entangled photons in our experiment are GaAs/ AlGaAs QDs embedded in a broadband optical antenna, offering photon extraction efficiencies up to $65 \%$ while preserving a high single-photon purity and entanglement fidelity [26].

At a sample temperature of $T=4 \mathrm{~K}$, a selected QD is first triggered by optically pumping the surrounding host semiconductor material. The emission spectrum in Fig. 2(b) displays two prominent features: the exciton $(\mathrm{X})$ emission at $780.0 \mathrm{~nm}$ and the biexciton (XX) emission at $781.6 \mathrm{~nm}$. Here, the $\mathrm{X}$ photons reside near the optical $\mathrm{D}_{2}$ transitions of rubidium, a prominent quantum memory candidate [30].

To generate polarization-entangled photon pairs, we exploit the XX-X radiative cascade [22]. Deterministic excitation of the XX state is ensured by resonant twophoton excitation [31]. Light from a pulsed Ti:sapphire laser is sent to a home-built pulse-shaping setup to excite the QD. A pair of photons is emitted in the successive decay via the intermediate $X$ states to the ground state [left inset of Fig. 2(c)]. The photons share the polarization-entangled Bell state $\left|\Phi^{+}\right\rangle_{i}$ in the respective emission $i=1,2$ :

$$
\left|\Phi^{+}\right\rangle_{i}=\frac{1}{\sqrt{2}}\left(\left|H_{\mathrm{X}} H_{\mathrm{XX}}\right\rangle+\left|V_{\mathrm{X}} V_{\mathrm{XX}}\right\rangle\right)
$$

with $H$ and $V$ representing horizontal and vertical polarization of the rectilinear basis.

Figure 2(c) shows the resonant two-photon excitation spectrum of the XX cascade emission. The resonant laser is well suppressed using notch filters. Autocorrelation measurements [32] show high single-photon purities for $\mathrm{X}$ and $\mathrm{XX}$ emissions of $g_{\mathrm{X}}^{(2)}(0)=0.0041 \pm 0.0003$ and $g_{\mathrm{XX}}^{(2)}(0)=$ $0.0050 \pm 0.0005$ (see inset).

By sending the excitation laser light into a tunable, unbalanced Mach-Zehnder interferometer [see Fig. 2(a)], the QD is triggered to emit two consecutive pairs of photons with a time delay of 2 ns. Emissions 1 and 2 are separated using a nonpolarizing beam splitter and timegated single-photon detection. The XX and $\mathrm{X}$ photons from each pair are split apart using dichroic optical filters.

At this stage, the four-photon state $|\alpha\rangle$ is a product of the states from emissions 1 and 2. It can be rewritten into products of Bell states between the $\mathrm{X}$ and $\mathrm{XX}$ photons:

$$
\begin{aligned}
|\alpha\rangle= & \left|\Phi^{+}\right\rangle_{1}\left|\Phi^{+}\right\rangle_{2} \\
= & \frac{1}{2}\left(\left|\Phi^{+}\right\rangle_{\mathrm{X}}\left|\Phi^{+}\right\rangle_{\mathrm{XX}}+\left|\Phi^{-}\right\rangle_{\mathrm{X}}\left|\Phi^{-}\right\rangle_{\mathrm{XX}}\right. \\
& \left.+\left|\Psi^{+}\right\rangle_{\mathrm{X}}\left|\Psi^{+}\right\rangle_{\mathrm{XX}}+\left|\Psi^{-}\right\rangle_{\mathrm{X}}\left|\Psi^{-}\right\rangle_{\mathrm{XX}}\right)
\end{aligned}
$$


with the four polarization Bell states being

$$
\begin{aligned}
& \left|\Phi^{ \pm}\right\rangle=\frac{1}{\sqrt{2}}(|H H\rangle \pm|V V\rangle), \\
& \left|\Psi^{ \pm}\right\rangle=\frac{1}{\sqrt{2}}(|H V\rangle \pm|V H\rangle) .
\end{aligned}
$$

Projecting $|\alpha\rangle$ to a Bell state between photons XX1 and XX2 will in turn result in a Bell state shared by the previously uncorrelated $\mathrm{X} 1$ and $\mathrm{X} 2$. We project to the state $\left|\Psi^{+}\right\rangle$[33] by performing the following BSM: First, photons $\mathrm{XX} 1$ and $\mathrm{XX} 2$ are sent to interfere on a nonpolarizing beam splitter. To ensure successful quantum interference, the arrival times of XX1 and XX2 are matched by delaying the XX1 photons before the BSM. The photons then pass through an $\mathrm{H}$ - or a $\mathrm{V}$-oriented polarizer in each beam splitter output, which reduces the heralding efficiency but ensures that photons in the wrong polarization mode are discarded [33]. Single-mode fibers deliver the photons to superconducting nanowire single-photon detectors (SNSPDs) with time resolutions of $50 \mathrm{ps.}$

Successful coincidence detection at the BSM now leaves the two remaining photons $\mathrm{X} 1$ and $\mathrm{X} 2$ in the Bell state

$$
\left|\Phi^{+}\right\rangle_{A B}=\frac{1}{\sqrt{2}}(|H V\rangle+|V H\rangle)
$$

sent to Alice and Bob for measurement. Subsequent arrangement of a quarter-wave plate, half-wave plate (HWP), polarizer, and SNSPD allows for projection on any desired polarization state.

Successful entanglement swapping relies on high entanglement fidelities $f_{i}$ of the initial photon pairs (emission $i=1,2$ ) and on high photon indistinguishabilities $I$ of the $\mathrm{XX}$ photons sent to the BSM. We perform quantum state tomography [42] to reconstruct the full two-photon density matrix $\rho_{i}$ of emissions $i=1,2$ as shown in Figs. 3(a) and 3(b), respectively. The real (left) and imaginary (right) parts clearly resemble the Bell state $\left|\Phi^{+}\right\rangle$. We obtain fidelities of $f_{1}=0.9369 \pm 0.0004$ (emission 1) and $f_{2}=$ $0.9267 \pm 0.0004$ (emission 2) indicating highly entangled photon emission.

Figure 3(c) shows a coincidence histogram obtained in an indistinguishability measurement [43] based on HongOu-Mandel interference [44]. The two consecutive XX photons are guided into a separate, unbalanced MachZehnder interferometer featuring a time delay identical to that between XX1 and XX2. Reduced coincidences are observed on two subsequent avalanche photodiodes with a time resolution of $>300 \mathrm{ps}$. The photon polarization is then changed by a HWP, rendering the photons distinguishable and increasing the coincidences. The coincidences for parallel polarizations (red) show a significant reduction in comparison with those for perpendicular polarizations (black). After correcting for imperfections of the optical
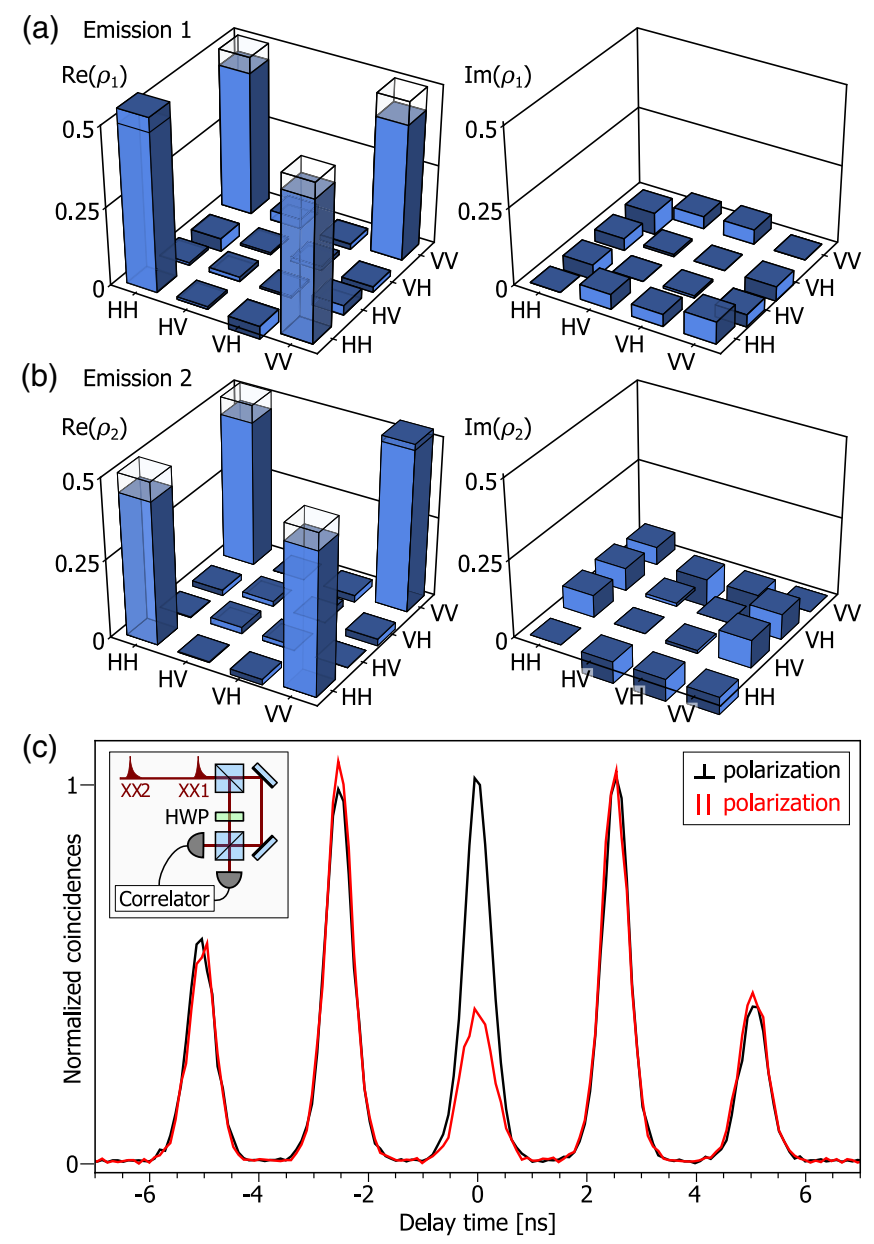

FIG. 3. Degree of entanglement and photon indistinguishability. Two-photon density matrices of the photon pairs $\mathrm{X} i-\mathrm{XX} i$ from (a) emission $i=1$ and (b) emission $i=2$, resembling the Bell state $\left|\Phi^{+}\right\rangle$(shaded areas) with fidelities $f_{i}$ of $f_{1}=0.9369 \pm$ 0.0004 and $f_{2}=0.9267 \pm 0.0004$. (c) The indistinguishability $I=0.569 \pm 0.009$ of photons XX1 and XX2 derived from a Hong-Ou-Mandel measurement using a separate, unbalanced Mach-Zehnder interferometer (inset) and avalanche photodiodes. By means of a HWP, copolarized photons yield reduced coincidences (red) compared with crossed polarizations (black). For entanglement swapping, the indistinguishability is further increased by time gating at the Bell state measurement.

setup [33], we extract photon indistinguishabilities of $I=0.569 \pm 0.009$, which directly specifies the success probability of the BSM in the entanglement swapping experiment. Time gating of BSM events further increases the photon indistinguishability.

As a control measurement, the photon state shared by Alice and Bob is first investigated without considering the BSM. The density matrix $\rho_{\text {mix }}$ extracted from our observations via quantum state tomography is shown in Fig. 4(a). The signature of a statistical mixture of polarization states is evident, with a fidelity of $f_{\text {mix }}=0.9960 \pm 0.0004$ to the completely mixed state $\frac{1}{4} \rrbracket$. This is expected since the photons $\mathrm{X} 1$ and $\mathrm{X} 2$ do not stem from the same emission cascade. 
(a) Without BSM (no swapping)

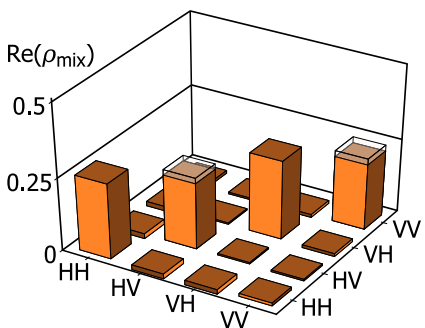

(c)

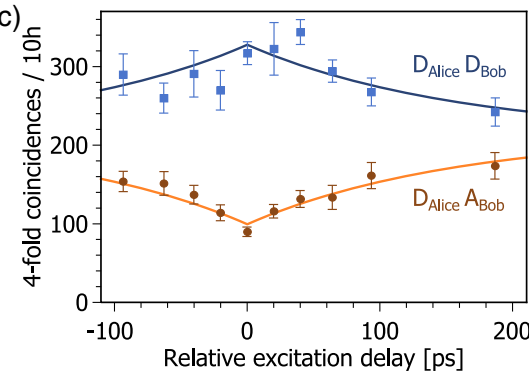

(b) With BSM (entanglement swapping)

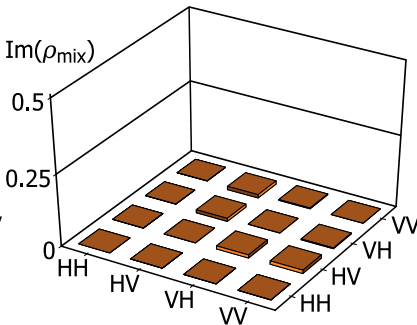

(d)

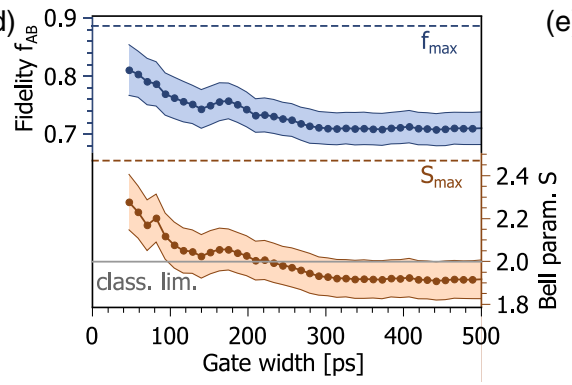

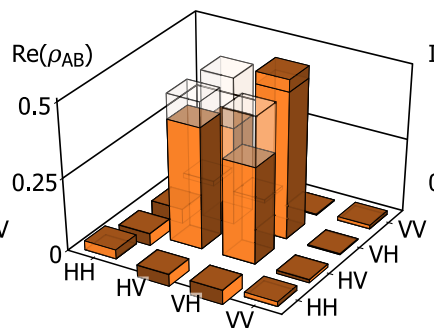

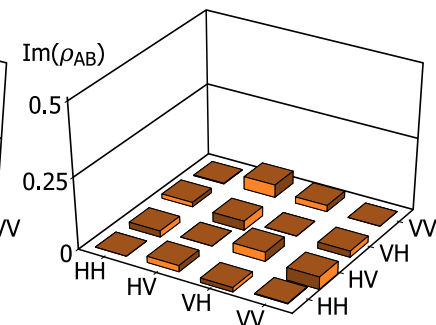

(e)

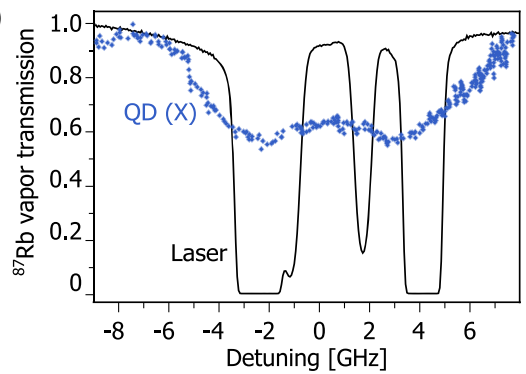

FIG. 4. Entanglement swapping with semiconductor-generated photons. Density matrix of the two-photon state received by Alice and Bob without (a) and with (b) a heralding BSM. The shaded areas represent the difference from the ideally obtainable values. The real part (left) and imaginary part (right) of $\rho_{\text {mix }}$ show the distinct signature of a perfect statistical mixture $\frac{1}{4} \mathbb{1}$, whereas $\rho_{A B}$ closely resembles the entangled state $\left|\Psi^{+}\right\rangle$with a fidelity of $f_{A B}=0.81 \pm 0.04$ (with time gating at BSM). (c) Fourfold coincidences as a function of the delay between photons XX1 and XX2 at the BSM (without time gating). Measurement settings of Alice and Bob in the copolarized (orange) and cross-polarized (blue) diagonal bases reveal a large difference at zero time delay, indicating successful entanglement swapping. The solid lines denote the double-sided exponential fit. (d) Fidelity $f$ and Bell parameter $S$ as a function of gate width of photon detection at the BSM. Large gate widths result in a decreased fidelity of $f_{A B}=0.71 \pm 0.03$. At $47 \mathrm{ps}$ gate width, $S=2.28 \pm 0.13$ is obtained, violating the $\mathrm{CHSH}$ and Bell inequalities. The dotted lines are the maximally achievable values in the case of perfect photon indistinguishability. (e) ${ }^{87} \mathrm{Rb}$ vapor cell transmission spectra at the $D_{2}$ transitions, measured with a narrow-band laser (black) and the frequency-detuned X photons from the QD (blue).

Now the entanglement is swapped from the initial photon pairs to the photons received by Alice and Bob, as established by coincidences at the BSM. In order to estimate the final state $\rho_{A B}$, we developed a theoretical model [33] that solely requires the density matrices $\rho_{1}$ and $\rho_{2}$ and the photon indistinguishability $I$. The final state $\rho_{A B}$ is written as the weighted sum of the expected density matrices $\rho_{A B}^{I=0}$ and $\rho_{A B}^{I=1}$ (derived in [33]) when assuming zero or unity indistinguishability:

$$
\rho_{A B}=I \rho_{A B}^{I=1}+(1-I) \rho_{A B}^{I=0} .
$$

Based on the measurement results obtained in Figs. 3(a) and 3(b), the fidelity to the Bell state $\left|\Psi^{+}\right\rangle$is expected to be $f_{A B}=0.71$. Distinguishable photons $(I=0)$ result in a fidelity as high as $f_{A B}=0.48$ [33] since photon pairs that are in the right spatial but wrong polarization mode are discarded by the polarizers in our BSM. We increase $f_{A B}$ further by postselecting BSM detection events that lie in a time gate width of $47 \mathrm{ps}$, at the expense of the total rate of heralding events. The ideal limit of $I=1$ (zero gate width) would result in a fidelity of $f_{A B}=0.89$.

Quantum state tomography is performed using sets of fourfold coincidences at different polarization settings for Alice and Bob. Each SNSPD in the setup detects approximately 0.5 million QD photons per second. The determined density matrix shown in Fig. 4 closely resembles the Bell state $\left|\Psi^{+}\right\rangle$. The fidelity of $f_{A B}=0.81 \pm 0.04$ clearly surpasses the classical limit of 0.5 and therefore testifies to the successful swapping of the entangled state. Based on our theoretical model, we estimate a photon indistinguishability of $I=0.823 \pm 0.017$ in that case.

Figure 4(c) features the measurement of fourfold coincidences in the copolarized and cross-polarized diagonal bases as a function of the relative time delay between emissions 1 and 2. In this fashion the temporal overlap of the XX photons at the beam splitter in the BSM is tuned. The highest XX photon indistinguishability is found at zero delay, resulting in a distinct coincidence offset for copolarized and cross-polarized bases. As the delay time departs from zero, the BSM success starts to drop. This results in assimilating fourfold coincidences. The data, obtained without time gating at the BSM, can be well fitted to double-sided exponential functions denoted as solid lines.

One bottleneck in reaching higher $f_{A B}$ is the $\mathrm{XX}$ photon indistinguishability, resulting in the deviant off-diagonal elements of $\operatorname{Re}\left(\rho_{A B}\right)$ [33]. In Fig. 4(d) the fidelity $f_{A B}$ and 
the Bell parameter $S$, as used in the CHSH Bell inequalities $[27,28]$, are plotted against the temporal gate width. In perfect agreement with our theoretical model, the fidelity decreases to $f_{A B}=0.71 \pm 0.03$ for large gate widths. Smaller gate widths result in values up to $f_{A B}=$ $0.81 \pm 0.04$. A further increase in the ideal fidelity of $f_{\max }=0.89$ [33] for gate width approaching zero is unattainable due to the time resolution of the detectors. The Bell parameter $S=2.28 \pm 0.13$ at the 47 ps gate violates the $\mathrm{CHSH}$ and Bell inequalities, $S \leq 2$, by more than 2 standard deviations. Assuming perfect indistinguishability, it reaches $S_{\max }=2.47$.

In a final step we investigate the compatibility of our semiconductor entangled photon source with atomic transitions of rubidium. Maintaining entangled photon emission, the emission frequency is tuned over the $\mathrm{Rb} D_{2}$ transitions at $780.04 \mathrm{~nm}$ by controlling the QD temperature [6]. Figure 4(e) displays the transmission of a heated ${ }^{87} \mathrm{Rb}$ vapor cell against the relative frequency detuning of a spectrally narrow laser (black). Two prominent absorption features are observed, corresponding to the two ${ }^{87} \mathrm{Rb}$ ground states split by the hyperfine interaction [45]. The transmission of the QD photons (blue) shows two clear absorption dips, which are broadened due to the QD linewidth of $\Delta \nu=(4.9 \pm$ $0.2) \mathrm{GHz}$. This opens the door for further experiments involving the storage of polarization-encoded qubits in atomic quantum memories for hybrid quantum repeaters. Efficient storage relies on linewidth matching of the hybrid system by using, e.g., lifetime-limited QD emission [23] and atomic ensembles [46] in microscopic fiber cavities [47]. In addition, $\mathrm{Rb}$ atomic transitions can serve as a common and global reference at which the QD emission can be frequency stabilized [48]. Thus, the indistinguishability of photons from distant nodes in a quantum network could be ensured.

In conclusion, we have realized the first entanglement swapping based on photons from a quantum dot, laying the foundations for scalable semiconductor based quantum networks. Therefore, the swapping of entanglement with photons from distant emitters or the realization of multiphoton entanglement comes into reach. For practical application in quantum communication systems, further efforts should be spent on improving the photon indistinguishability, entanglement fidelity, and source brightness. These stringent requirements can be achieved, e.g., by integrating quantum dots into microcavities $[49,50]$. Applying electric fields in QD integrated diode structures may further decrease photon dephasing [51]. Furthermore, the indistinguishability of photons from distant emitters can be ensured using integrated straintuning platforms [52].

We acknowledge funding by the BMBF (Q.com) and the European Research Council (QD-NOMS). F. D. acknowledges support by the IFW Excellence Program. We thank Wenjamin Rosenfeld (LMU München), Tobias Macha (Universität Bonn), Matthew T. Eiles (MPIPKS
Dresden), and Franz J. F. Löchner (FSU Jena) for fruitful discussions.

Note added.-Recently, we noticed a similar work appearing as an e-print [38].

*f.ding@fkp.uni-hannover.de o.schmidt@ifw-dresden.de

[1] R. N. Hall, G. E. Fenner, J. D. Kingsley, T. J. Soltys, and R. O. Carlson, Coherent Light Emission from GaAs Junctions, Phys. Rev. Lett. 9, 366 (1962).

[2] H. Kroemer, A proposed class of hetero-junction injection lasers, Proc. IEEE 51, 1782 (1963).

[3] I. Melngailis, Longitudinal injection-plasma laser of InSb, Appl. Phys. Lett. 6, 59 (1965).

[4] H. Soda, K. i. Iga, C. Kitahara, and Y. Suematsu, GaInAsP/ InP surface emitting injection lasers, Jpn. J. Appl. Phys. 18, 2329 (1979).

[5] N. Somaschi, V. Giesz, L. De Santis, J. C. Loredo, M. P. Almeida, G. Hornecker, S. L. Portalupi, T. Grange, C. Antón, J. Demory, C. Gómez, I. Sagnes, N. D. LanzillottiKimura, A. Lemaítre, A. Auffeves, A. G. White, L. Lanco, and P. Senellart, Near-optimal single-photon sources in the solid state, Nat. Photonics 10, 340 (2016).

[6] X. Ding, Y. He, Z.-C. Duan, N. Gregersen, M.-C. Chen, S. Unsleber, S. Maier, C. Schneider, M. Kamp, S. Höfling, C.-Y. Lu, and J.-W. Pan, On-Demand Single Photons with High Extraction Efficiency and Near-Unity Indistinguishability from a Resonantly Driven Quantum Dot in a Micropillar, Phys. Rev. Lett. 116, 020401 (2016).

[7] C. L. Salter, R. M. Stevenson, I. Farrer, C. A. Nicoll, D. A. Ritchie, and A.J. Shields, An entangled-light-emitting diode, Nature (London) 465, 594 (2010).

[8] N. Gisin, G. Ribordy, W. Tittel, and H. Zbinden, Quantum cryptography, Rev. Mod. Phys. 74, 145 (2002).

[9] J. P. Dowling, Quantum optical metrology-The lowdown on high-N00N states, Contemp. Phys. 49, 125 (2008).

[10] E. Knill, R. Laflamme, and G. J. Milburn, A scheme for efficient quantum computation with linear optics, Nature (London) 409, 46 (2001).

[11] H. J. Kimble, The quantum internet, Nature (London) 453 , 1023 (2008).

[12] S. Bose, V. Vedral, and P. L. Knight, Multiparticle generalization of entanglement swapping, Phys. Rev. A 57, 822 (1998).

[13] H.-J. Briegel, W. Dür, J. I. Cirac, and P. Zoller, Quantum Repeaters: The Role of Imperfect Local Operations in Quantum Communication, Phys. Rev. Lett. 81, 5932 (1998).

[14] C. H. Bennett, G. Brassard, C. Crépeau, R. Jozsa, A. Peres, and W. K. Wootters, Teleporting an Unknown Quantum State via Dual Classical and Einstein-Podolsky-Rosen Channels, Phys. Rev. Lett. 70, 1895 (1993).

[15] M. Żukowski, A. Zeilinger, M. A. Horne, and A. K. Ekert, "Event-Ready-Detectors" Bell Experiment via Entanglement Swapping, Phys. Rev. Lett. 71, 4287 (1993).

[16] J.-W. Pan, D. Bouwmeester, H. Weinfurter, and A. Zeilinger, Experimental Entanglement Swapping: Entangling Photons that Never Interacted, Phys. Rev. Lett. 80, 3891 (1998). 
[17] P. G. Kwiat, K. Mattle, H. Weinfurter, A. Zeilinger, A. V. Sergienko, and Y. Shih, New High-Intensity Source of Polarization-Entangled Photon Pairs, Phys. Rev. Lett. 75, 4337 (1995).

[18] Y. H. Shih and C. O. Alley, New Type of Einstein-PodolskyRosen-Bohm Experiment Using Pairs of Light Quanta Produced by Optical Parametric Down Conversion, Phys. Rev. Lett. 61, 2921 (1988).

[19] E. Meyer-Scott, N. Prasannan, C. Eigner, V. Quiring, J. M. Donohue, S. Barkhofen, and C. Silberhorn, Highperformance source of spectrally pure, polarization entangled photon pairs based on hybrid integrated-bulk optics, Opt. Express 26, 32475 (2018).

[20] X.-L. Wang, L.-K. Chen, W. Li, H.-L. Huang, C. Liu, C. Chen, Y.-H. Luo, Z.-E. Su, D. Wu, Z.-D. Li, H. Lu, Y. Hu, X. Jiang, C.-Z. Peng, L. Li, N.-L. Liu, Y.-A. Chen, C.-Y. Lu, and J.-W. Pan, Experimental Ten-Photon Entanglement, Phys. Rev. Lett. 117, 210502 (2016).

[21] V. Scarani, H. de Riedmatten, I. Marcikic, H. Zbinden, and N. Gisin, Four-photon correction in two-photon Bell experiments, Eur. Phys. J. D 32, 129 (2005).

[22] N. Akopian, N. H. Lindner, E. Poem, Y. Berlatzky, J. Avron, D. Gershoni, B. D. Gerardot, and P. M. Petroff, Entangled Photon Pairs from Semiconductor Quantum Dots, Phys. Rev. Lett. 96, 130501 (2006).

[23] J.-P. Jahn, M. Munsch, L. Béguin, A. V. Kuhlmann, M. Renggli, Y. Huo, F. Ding, R. Trotta, M. Reindl, O. G. Schmidt, A. Rastelli, P. Treutlein, and R. J. Warburton, An artificial $\mathrm{Rb}$ atom in a semiconductor with lifetime-limited linewidth, Phys. Rev. B 92, 245439 (2015).

[24] R. Keil, M. Zopf, Y. Chen, B. Höfer, J. Zhang, F. Ding, and O. G. Schmidt, Solid-state ensemble of highly entangled photon sources at rubidium atomic transitions, Nat. Commun. 8, 15501 (2017).

[25] D. Huber, M. Reindl, Y. Huo, H. Huang, J. S. Wildmann, O. G. Schmidt, A. Rastelli, and R. Trotta, Highly indistinguishable and strongly entangled photons from symmetric GaAs quantum dots, Nat. Commun. 8, 15506 (2017).

[26] Y. Chen, M. Zopf, R. Keil, F. Ding, and O. G. Schmidt, Highly-efficient extraction of entangled photons from quantum dots using a broadband optical antenna, Nat. Commun. 9, 2994 (2018).

[27] J. S. Bell, On the Einstein Podolsky Rosen paradox, Phy. Physique Fiz. 1, 195 (1964).

[28] J. F. Clauser, M. A. Horne, A. Shimony, and R. A. Holt, Proposed Experiment to Test Local Hidden-Variable Theories, Phys. Rev. Lett. 23, 880 (1969).

[29] M. T. Rakher, R. J. Warburton, and P. Treutlein, Prospects for storage and retrieval of a quantum-dot single photon in an ultracold ${ }^{87} \mathrm{Rb}$ ensemble, Phys. Rev. A 88, 053834 (2013).

[30] M. Körber, O. Morin, S. Langenfeld, A. Neuzner, S. Ritter, and G. Rempe, Decoherence-protected memory for a singlephoton qubit, Nat. Photonics 12, 18 (2018).

[31] M. Müller, S. Bounouar, K. D. Jöns, M. Glässl, and P. Michler, On-demand generation of indistinguishable polarization-entangled photon pairs, Nat. Photonics 8, 224 (2014).

[32] R. Hanbury Brown and R. Q. Twiss, A test of a new type of stellar interferometer on Sirius, Nature (London) 178, 1046 (1956).
[33] See Supplemental Material at http://link.aps.org/ supplemental/10.1103/PhysRevLett.123.160502 for details and supporting measurements, which includes Refs. [16,34-41].

[34] V. Giesz, S. L. Portalupi, T. Grange, C. Antón, L. De Santis, J. Demory, N. Somaschi, I. Sagnes, A. Lemaître, L. Lanco, A. Auffèves, and P. Senellart, Cavity-enhanced two-photon interference using remote quantum dot sources, Phys. Rev. B 92, 161302(R) (2015).

[35] R.-B. Jin, M. Takeoka, U. Takagi, R. Shimizu, and M. Sasaki, Highly efficient entanglement swapping and teleportation at telecom wavelength, Sci. Rep. 5, 9333 (2015).

[36] R. Valivarthi, M. G. Puigibert, Q. Zhou, G. H. Aguilar, V. B. Verma, F. Marsili, M. D. Shaw, S. W. Nam, D. Oblak, and W. Tittel, Quantum teleportation across a metropolitan fibre network, Nat. Photonics 10, 676 (2016).

[37] Y. Zhang, M. Agnew, T. Roger, F. S. Roux, T. Konrad, D. Faccio, J. Leach, and A. Forbes, Simultaneous entanglement swapping of multiple orbital angular momentum states of light, Nat. Commun. 8, 632 (2017).

[38] F. Basso Basset, M. B. Rota, C. Schimpf, D. Tedeschi, K. D. Zeuner, S. F. C. da Silva, M. Reindl, V. Zwiller, K. D. Jöns, A. Rastelli, and R. Trotta, preceding Letter, Entanglement Swapping with Photons Generated on-Demand by a Quantum Dot, Phys. Rev. Lett. 123, 160501 (2019).

[39] J. Nilsson, R. M. Stevenson, K. H. A. Chan, J. SkibaSzymanska, M. Lucamarini, M. B. Ward, A. J. Bennett, C. L. Salter, I. Farrer, D. A. Ritchie, and A. J. Shields, Quantum teleportation using a light-emitting diode, Nat. Photonics 7, 311 (2013).

[40] E. Megidish, A. Halevy, T. Shacham, T. Dvir, L. Dovrat, and H. S. Eisenberg, Entanglement Swapping Between Photons that Have Never Coexisted, Phys. Rev. Lett. 110, 210403 (2013).

[41] J.-W. Pan, M. Daniell, S. Gasparoni, G. Weihs, and A. Zeilinger, Experimental Demonstration of Four-Photon Entanglement and High-Fidelity Teleportation, Phys. Rev. Lett. 86, 4435 (2001).

[42] D. F. V. James, P. G. Kwiat, W. J. Munro, and A. G. White, Measurement of qubits, Phys. Rev. A 64, 052312 (2001).

[43] C. Santori, D. Fattal, J. Vučković, G. S. Solomon, and Y. Yamamoto, Indistinguishable photons from a single-photon device, Nature (London) 419, 594 (2002).

[44] C. K. Hong, Z. Y. Ou, and L. Mandel, Measurement of Subpicosecond Time Intervals Between Two Photons by Interference, Phys. Rev. Lett. 59, 2044 (1987).

[45] S. Penselin, T. Moran, V. W. Cohen, and G. Winkler, Hyperfine structure of the electronic ground states of $\mathrm{Rb}^{85}$ and $\mathrm{Rb}^{87}$, Phys. Rev. 127, 524 (1962).

[46] R. J. Thompson, G. Rempe, and H. J. Kimble, Observation of Normal-Mode Splitting for an Atom in an Optical Cavity, Phys. Rev. Lett. 68, 1132 (1992).

[47] T. Macha, E. Uruñuela, W. Alt, M. Ammenwerth, D. Pandey, H. Pfeifer, and D. Meschede, Non-adiabatic storage of short light pulses in an atom-cavity system, arXiv:1903 .10922 .

[48] M. Zopf, T. Macha, R. Keil, E. Uruñuela, Y. Chen, W. Alt, L. Ratschbacher, F. Ding, D. Meschede, and O. G. Schmidt, 
Frequency feedback for two-photon interference from separate quantum dots, Phys. Rev. B 98, 161302(R) (2018).

[49] P. Senellart, G. Solomon, and A. White, High-performance semiconductor quantum-dot single-photon sources, Nat. Nanotechnol. 12, 1026 (2017).

[50] L. Sapienza, M. Davanço, A. Badolato, and K. Srinivasan, Nanoscale optical positioning of single quantum dots for bright and pure single-photon emission, Nat. Commun. 6, 7833 (2015).
[51] A. V. Kuhlmann, J. Houel, A. Ludwig, L. Greuter, D. Reuter, A. D. Wieck, M. Poggio, and R. J. Warburton, Charge noise and spin noise in a semiconductor quantum device, Nat. Phys. 9, 570 (2013).

[52] Y. Chen, J. Zhang, M. Zopf, K. Jung, Y. Zhang, R. Keil, F. Ding, and O. G. Schmidt, Wavelength-tunable entangled photons from silicon-integrated III-V quantum dots, Nat. Commun. 7, 10387 (2016). 